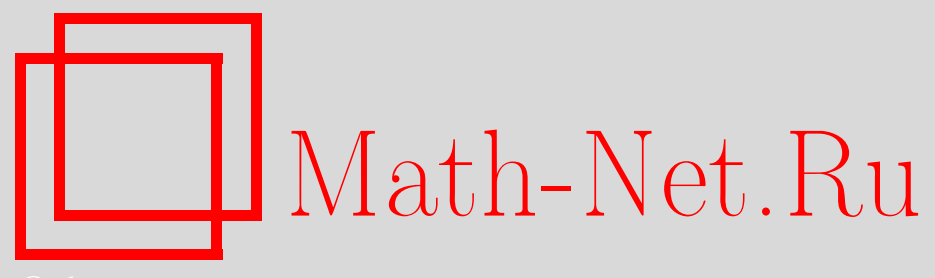

А. Заславский, Об одной ортоцентрической четверке, Квант, 2019, номер 7, 20-23

DOI: https://doi.org/10.4213/kvant20190703

Использование Общероссийского математического портала Math-Net.Ru подразумевает, что вы прочитали и согласны с пользовательским соглашением http://www.mathnet.ru/rus/agreement

Параметры загрузки:

IP : 54.89 .56 .158

26 апреля 2023 г., 17:06:46 


\section{Об одной ортоцентрической четверке}

\section{А.ЗАСЛАВСКИЙ}

Эта статья посвящена геометрической конструкции из задачи М2553 «Задачника «Кванта» (эта задача также предлагалась на XXII Кубке памяти А.Н.Колмогорова). В процессе изучения этой конструкции, а также совместной работы над ней вместе с И.Богдановым и П.Кожевниковым обнаружилось довольно много интересных геометрических сюжетов, о которых мы и хотим рассказать читателям. Но начнем с самой исходной задачи.

Задача М2553*. Обозначим через I центр окружности, вписанной в остроугольный треугольник $A B C$, через $A_{1}, B_{1}$ u $C_{1}-$ точки ее касания со сторонами $B C$, CA и АВ соответственно, через $K$ и L чентры окружностей, вписанных в четырехугольники $A B_{1} I C_{1}$ и $B A_{1} I C_{1}$ соответственно, через $Н$ - основание высоты,

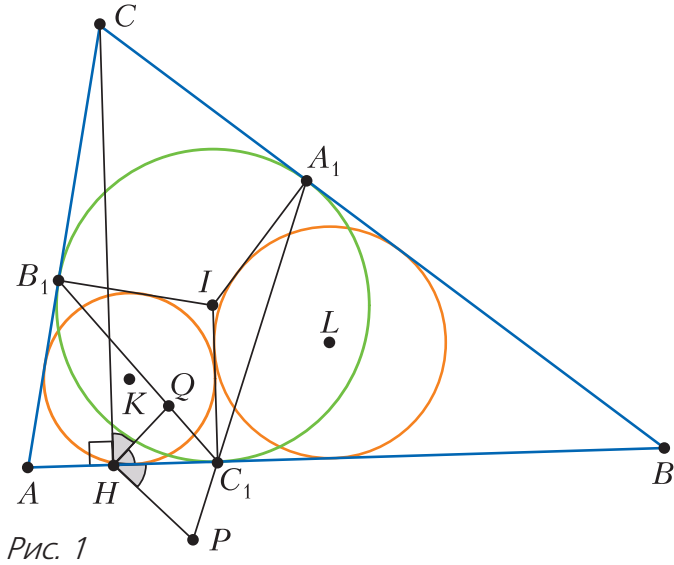

опущенной из точки С (рис.1). Биссектриса угла АНС пересекает прямую $A_{1} C_{1}$ в точке $P$, а биссектриса угла ВНС пересекает прямую $B_{1} C_{1}$ в точке $Q$. Докажите, что $Q$ - орточентр треугольника KLP.

Условие задачи, очевидно, означает, что каждая из точек $K, L, P, Q$ является ортоцентром треугольника, образованного тремя остальными. Такая четверка точек называется ортоцентрической.

DOI: https://doi.org/10.4213/kvant20190703
Ниже для краткости будем называть две фигуры перпендикулярно подобными, если они подобны, одинаково ориентированы и углы между соответствующими отрезками фигур равны $90^{\circ}$. Иными словами, называем фигуры перпендикулярно подобными, если одну из другой можно получить поворотной гомотетией (т.е. последовательным выполнением поворота и гомотетии) с углом поворота $90^{\circ}$.

Решение задачи. В случае $A C=B C$ точки $P, Q$ и $C_{1}$ совпадают, а треугольник $K C_{1} L$ - прямоугольный и равнобедренный, следовательно, утверждение задачи верно. Далее не умаляя общности считаем, что $A C<B C$.

Из симметрии четырехугольника $B A_{1} I C_{1}$ относительно $B I$ (рис.2) легко видеть, что

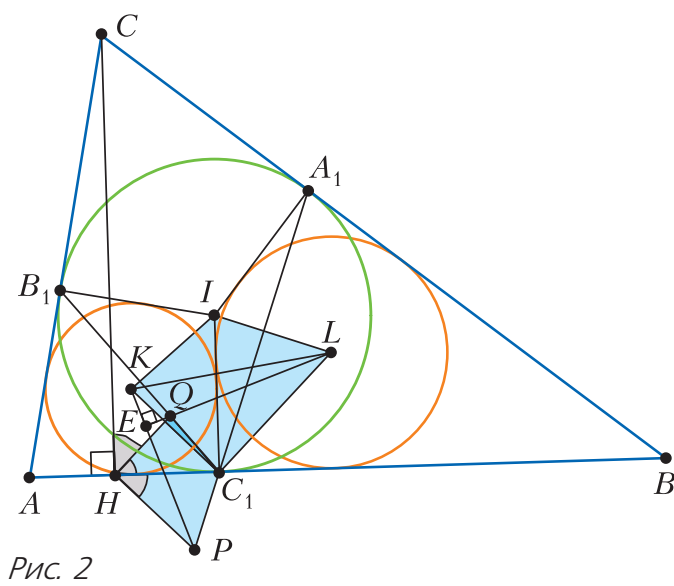

прямая $C_{1} A_{1}$ (на которой также лежит $P$ ) перпендикулярна прямой $B I$ (на которой также лежит $L$ ). Аналогично, перпендикулярны прямые $C_{1} Q B_{1}$ и $A K I$. Далее, поскольку $C_{1} K$ и $C_{1} L$ - биссектрисы прямых углов $A C_{1} I$ и $B C_{1} I$, имеем $P H\left\|C_{1} K \perp C_{1} L\right\| Q H$. Поэтому треугольники $K I C_{1}$ и $Q C_{1} H$ перпендикулярно подобны. Также перпендикулярно подобны треугольники $L I C_{1}$ и $C_{1} P H$, а значит, то же верно и для четырехугольников $K I L C_{1}$ и $Q C_{1} P H$, откуда $P Q$ и $K L$ перпендикулярны как соответствующие диагонали перпендикулярно подобных четырехугольников.

Теперь достаточно показать, что $P K \perp Q L$. Пусть $X=H P \cap A I$ и $Y=H Q \cap$ 


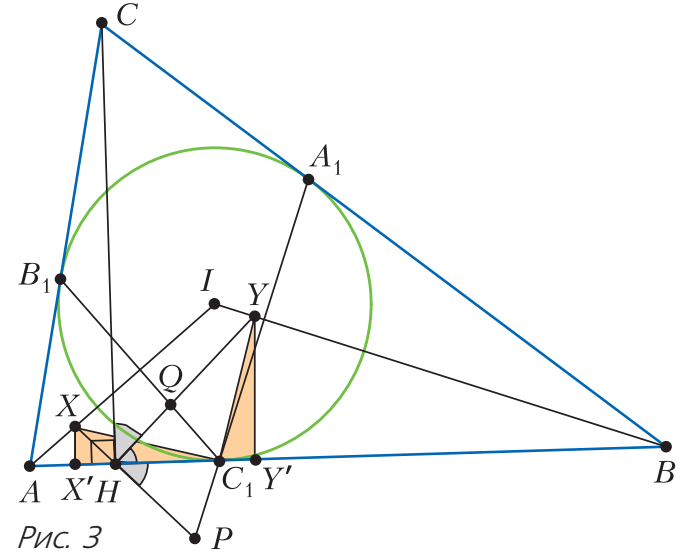

$\bigcap B I$ - центры окружностей, вписанных в треугольники $A H C, B H C$ (рис.3). Нетрудно показать, что $X C_{1} \perp Y C_{1}$ и $X C_{1}=Y C_{1}$. Действительно, если $X^{\prime}, Y^{\prime}$ - проекции $X, Y$ на $A B$, то $X X^{\prime}=C_{1} Y^{\prime}$ и $Y Y^{\prime}=C_{1} X^{\prime}$ (см. упражнение 1 ниже), а значит, прямоугольные треугольники $X X^{\prime} C_{1}$ и $C_{1} Y^{\prime} Y$ равны.

Упражнение 1. Докажите, что $X X^{\prime}=C_{1} Y^{\prime}$.

Указание. Из прямоугольного треугольника $A H C$ получаем $X X^{\prime}=X^{\prime} H$, а $C_{1} Y^{\prime}=B C_{1}-B Y^{\prime}$. Далее отрезки $X^{\prime} H, B C_{1}, B Y^{\prime}$ можно выразить как отрезки касательных до точки касания с вписанной окружностью в треугольниках $A H C$, $B H C, A B C$.

Из перпендикулярности $X C_{1}$ и $Y C_{1}$ получаем, что треугольники $P X C_{1}$ и $L C_{1} Y$ перпендикулярно подобны и даже равны (рис.4). Также перпендикулярно подобны треугольники $K X C_{1}$ и $Q C_{1} Y$, а значит, то же верно и для четырехугольников (трапеций) $P X K C_{1}$ и $L C_{1} Q Y$, откуда $P K \perp L Q$, что завершает решение.

Итак, исходная задача решена. Однако рассматриваемая конфигурация обладает рядом других красивых свойств.

Укажем некоторые интересные описания оснований высот нашей ортоцентрической четверки $K, L, P, Q$; назовем эти точки $D=K Q \cap L P, E=L Q \cap K P, F=K L \cap P Q$.

\section{Точка $\boldsymbol{F}$}

Из ортоцентрической четверки $K, L, P$, $Q$ имеем подобие $\triangle K F P \sim \triangle Q F L \quad$ (см. рис. 4$)$, иначе говоря, $F-$ центр поворотной гомотетии, переводящей вектор

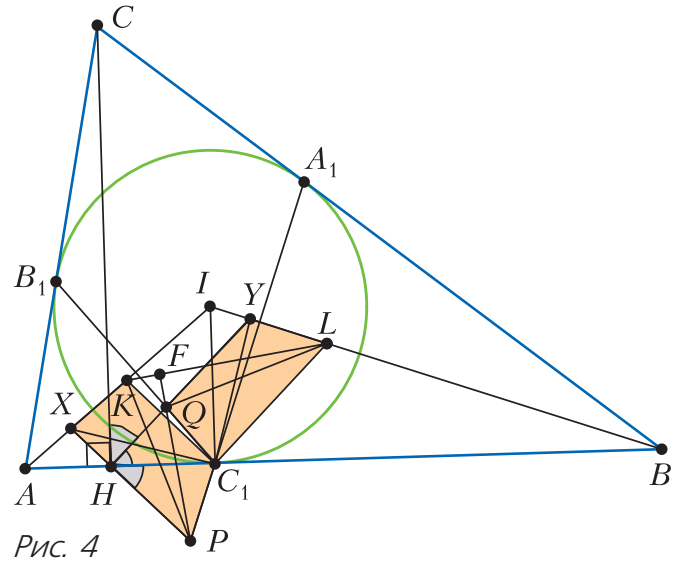

$\overrightarrow{K P}$ в $\overrightarrow{Q L}$. Эта поворотная гомотетия совмещает диагонали перпендикулярно подобных и равных трапеций $P X K C_{1}$ и $L C_{1} Q Y$. Значит, на самом деле эта поворотная гомотетия - просто поворот (на $\left.90^{\circ}\right)$, который, в частности, переводит $\overrightarrow{X C_{1}}$ в $\overrightarrow{C_{1} Y}$. Центр такого поворота середина гипотенузы $X Y$ прямоугольного равнобедренного треугольника $X C_{1} Y$. Тем самым, $F$ - середина $X Y$.

Кроме того, мы поняли, что треугольники $K F P$ и $Q F L$ не только подобны, но и равны, в частности $K P=Q L$. Из этого же равенства треугольников вытекает, что треугольник $P F L$ - равнобедренный, но, как мы знаем, он прямоугольный, откуда $\angle K L P=45^{\circ}$.

\section{Упражнения}

2. Докажите, что для любой ортоцентрической четверки $K, L, P, Q$ условие $K P=Q L$ эквивалентно равенству $45^{\circ}$ или $180^{\circ}+45^{\circ}$ любого из углов (невыпуклого) четырехугольника $K L P Q$.

3. Докажите, что $F$ лежит на серединном перпендикуляре к отрезку $H C_{1}$.

Указание. Можно использовать равенство $X^{\prime} H=Y^{\prime} C_{1}$ (см. упражнение 1$)$.

\section{Точка $E$}

Пользуясь нашей ортоцентрической четверкой, понимаем, что $E$ - центр поворотной гомотетии, переводящей вектор $\overrightarrow{P Q}$ в $\overrightarrow{L K}$ (см. рис.2). Эта поворотная гомотетия совмещает диагонали перпендикулярно подобных четырехугольников $Q C_{1} P H$ и $K I L C_{1}$. Значит, это поворотная гомотетия (с углом поворота $90^{\circ}$ ), которая пере- 
водит $\overrightarrow{H C_{1}}$ в $\overrightarrow{C_{1} I}$. Центр такой поворотной гомотетии - проекция вершины $C_{1}$ на гипотенузу $I H$ прямоугольного треугольника $I C_{1} H$. Таким образом, $E$ совпадает с проекцией $C_{1}$ на $I H$.

\section{Точка $D$}

Так как $P H \perp Q H$ и $P D \perp D Q$, точка $D$ лежит на окружности (PHQ) (рис.5).

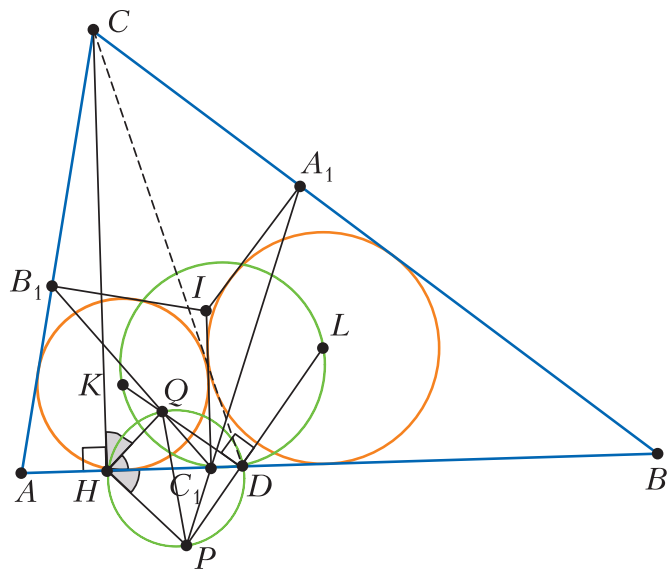

Pис. 5

Упражнение 4. Докажите, что $E$ также лежит на окружности $(P H Q)$.

Как мы знаем, треугольник $P D Q$ - прямоугольный равнобедренный, $D$ - середина дуги $P Q$, поэтому $H D$ - биссектриса угла $P H Q$. Значит, прямая $H D$ совпадает с $A B$, т.е. $D$ лежит на $A B$.

Отметим, что $D$ также лежит на окружности $\left(K C_{1} L\right)$ с диаметром $K L$. Таким образом, $D$ - точка, в которой окружность $\left(K L C_{1}\right)$ вторично пересекает $A B$.

Известно (см., например, статью «Описанные четырехугольники и ломаные» в «Кванте» №1 за 2010 г., задача 3), что общая внутренняя касательная к окружностям, вписанным в четырехугольники $A B_{1} I C_{1}$ и $B A_{1} I C_{1}$, отличная от $I C_{1}$, проходит через $C$. Пусть эта касательная $-C D^{\prime}$, где $D^{\prime}$ - точка на прямой $A B$. Тогда $C_{1}$ и $D^{\prime}$ лежат на окружности с диаметром $K L$, поскольку $K D^{\prime} \perp L D^{\prime}$ (биссектрисы углов между прямыми $C D^{\prime}$ и $\left.A B\right)$ и, аналогично, $K C_{1} \perp L C_{1}$ (биссектрисы углов между прямыми $I C_{1}$ и $A B$ ). Тогда $D^{\prime}$ совпадает с $D$.

\section{Упражнения}

5. Из полученных описаний точки $D$ выведите, что прямые $C D, I C_{1}$ и $K L$ пересекаются в одной точке.

Указание. Эти прямые - общие касательные и линия центров окружностей.

6. Докажите другим способом, используя теорему Дезарга, что а) $D$ лежит на $A B$, б) $E$ лежит на $I H$.

Указание. Можно применить теорему Дезарга к треугольникам а) $K L C_{1}$ и $Q P H$, б) $Q Y L$ и $P X K$.

Наконец, заметим еще один факт о точках $P$ и $Q$ : прямая $P Q$ проходит через $C$. Доказать это можно, например следующим образом, используя гармонические четверки. Рассмотрим точки $U$ и $V$ пересечения $\mathrm{CH}$ с $B_{1} C_{1}$ и $A_{1} C_{1}$ соответственно (рис.6). Нетрудно доказать (например,

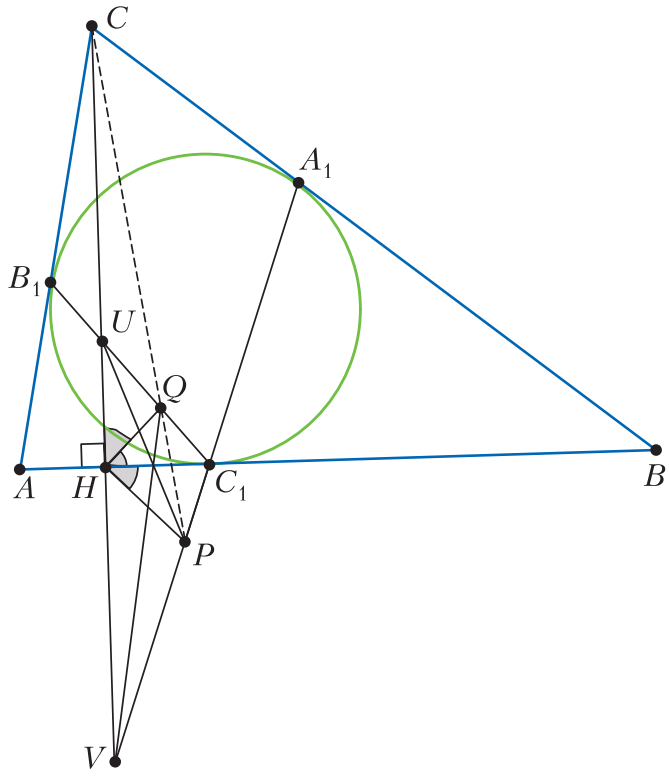

Pиc. 6

пользуясь теоремой Чевы для треугольника $\left.C_{1} U V\right)$, что прямые $U P, V Q$ и $C_{1} H$ пересекаются в одной точке, следовательно, $U, V, H$ и точка $P Q \cap C H$ образуют гармоническую четверку. Но прямые $C_{1} B_{1}$, $C_{1} A_{1}, C_{1} H$ и $C_{1} C$ также образуют гармоническую четверку, значит, точка пересечения $P Q \cap C H$ совпадает с $C$.

Упражнение 7. Докажите, что прямые $A H$ и $A D$ симметричны относительно $A F$.

Указание. Используйте упражнение 5. 
Подведем итог, еще раз сформулировав основные свойства данной конфигурации.

1. Точки $K, L, P, Q$ образуют ортоцентрическую четверку.

2. $K P=Q L ;$ углы четырехугольника $K L P Q$ равны $45^{\circ}$ или $180^{\circ}+45^{\circ}$.

3. Точка $F=K L \cap P Q-$ середина отрезка $X Y$.

4. Точка $E=L Q \cap K P-$ проекция $C_{1}$ на прямую $I H$.

5. Точка $D=K Q \cap L P$ лежит на прямой $A B$.

6. Прямая $P Q$ проходит через $C$.

В завершение скажем, что на XXII Кубке памяти А.Н.Колмогорова одиннадцатиклассник Владимир Петров из СанктПетербурга придумал другое решение исходной задачи, позволяющее обнаружить еще ряд свойств. Приведем план этого решения.

Так же, как и ранее, заметим перпендикулярное подобие четырехугольников $K I L C_{1}$ и $Q C_{1} P H$, откуда $P Q \perp K L$.
Пусть $E^{\prime}$ - проекция $C_{1}$ на $I H$ (по факту мы знаем, что $E^{\prime}=E$ ), тогда $E^{\prime}$ - центр поворотной гомотетии (с углом поворота $90^{\circ}$ ), переводящей $\overrightarrow{H C_{1}}$ в $\overrightarrow{C_{1} I}$, а значит, $Q C_{1} P H$ в $K I L C_{1}$. Из поворотной гомотетии $E^{\prime} P \perp E^{\prime} L$. Теперь достаточно доказать, что $\angle K E^{\prime} L=90^{\circ}$, т.е. точки $K, C_{1}$, $E^{\prime}, L$ лежат на одной окружности.

При инверсии относительно вписанной окружности $E^{\prime}$ переходит в $H$. Отметим точку $T$ на продолжении $H C$ за точку $C$ такую, что $C T=C B_{1}=C A_{1}$. Тогда для завершения решения достаточно доказать, что образы $K^{\prime}, L^{\prime}, H$ и $C_{1}$ точек $K, L, E^{\prime}$ и $C_{1}$ лежат на окружности с диаметром $C_{1} T$.

\section{Упражнения}

8. Докажите это.

9. Пусть окружность с центром $C$ и радиусом $C A_{1}=C B_{1}$ пересекает $C H$ в точке $G$. Докажите, что $F$ - середина $G C_{1}$.

\section{Юбилей теоремы Райского}

\section{(Начало см. на с. 10)}

отмеченных шестиугольников, а расстояние $d_{2}=1$ не реализуется на множествах $A_{5}$ и $A_{6}$ одинаково отмеченных треугольников.

\section{Ожидаемые продвижения}

Вопрос о том, можно ли аналогичным образом разбить плоскость на 4 или 5 множеств, остается открытым. Но недавнее доказательство [3] теоремы о том, что при раскраске плоскости в четыре цвета найдутся две точки одного цвета на расстоянии 1 , дает основание надеяться, что для четырех цветов утверждение теоремы Райского тоже верно. Достаточно найти дистанционный граф (т.е. граф, вершины которого - точки на плоскости, а ребрами соединены только пары вершин на расстоянии 1) из $n$ вершин, для которого, если выбрать не менее $n / 4$ вершин, какая-то пара выбранных вершин обязательно будет соединена ребром. Предлагаю читателям убедиться, что если в качестве зонда выбрать такой граф, то конструкция Райского доказывает утверждение для четырех цветов, а математикам-программистам - заняться увлекательной задачей поиска подходящего графа. Сейчас известно много графов, доказывающих теорему о четыpex цветах, но, возможно, не каждый граф, найденный для этой цели, обладает нужным нам свойством.

\section{Литература}

1. Д.Е.Райский. Реализация всех расстояний при разбиении пространства $\mathbb{R}^{n}$ на $n+1$ часть. Математические заметки, т.7, №3, 1970, с.319323.

2. Г.Хадвигер, Г.Дебруннер. Комбинаторная геометрия плоскости. - М., 1965.

3. А.Райгородский, В.Воронов, А.Савватеев, Прорыв в задаче о раскраске плоскости. - «Квант», 2018, №11. 